\title{
Preisach Modelling of Lithium-Iron-Phosphate Battery Hysteresis
}

\author{
Federico Baronti $^{\mathrm{a}}$, Nicola Femia ${ }^{\mathrm{c}}$, Roberto Saletti ${ }^{\mathrm{a}}$, Ciro Visone ${ }^{\mathrm{b}}$, Walter \\ Zamboni ${ }^{\mathrm{c}, *}$ \\ ${ }^{a}$ Dipartimento di Ingegneria dell'Informazione, Università di Pisa - Via Girolamo Caruso \\ 16, I-56126 Pisa, Italy \\ ${ }^{b}$ Dipartimento di Ingegneria, Università degli Studi del Sannio - Piazza Roma 21, I-82100 \\ Benevento, Italy \\ ${ }^{c}$ Dipartimento di Ingegneria dell'Informazione, Ingegneria Elettrica e Matematica Applicata \\ (DIEM), Università degli Studi di Salerno - Via Giovanni Paolo II 132, I-84084 Fisciano, \\ $S A$, Italy
}

\begin{abstract}
The hysteresis of the open-circuit voltage as a function of the state-of-charge in a 20 Ah lithium-iron-phosphate battery is investigated starting from pulsedcurrent experiments at a fixed temperature and ageing state, in order to derive a model that may reproduce well the battery behaviour. The hysteretic behaviour is modelled with the classical Preisach model used in magnetic materials. The paper shows that the Preisach model can successfully be applied to the lithium-ion battery hysteresis. First, the model is discretised by using the Everett function and identified by means of experiments, in which first-order reversal branches are measured. Then, the model is simulated and compared to some experimental data collected with different current profiles and to a one-state variable model previously used in the literature. The results show that the hysteresis is well reproduced with rms errors around $2 \%$. The advantages of the Preisach-based method, when compared to other models, are the formal and repeatable identification procedure and the limited computational resources needed for the model simulation that makes it appropriate for the online implementation on low-complexity hardware platforms.
\end{abstract}

\footnotetext{
*Corresponding author

Email address: wzamboni@unisa.it; tel. +39-089-963462; fax +39-089-968783 (Walter Zamboni)
} 
Keywords: Lithium-Iron-Phosphate $\left(\mathrm{LiFePO}_{4}\right)$ batteries, Hysteresis modelling, classical Preisach model, Open Circuit Voltage, State-of-Charge

\section{Introduction}

The effective storage of electric energy is becoming a challenge that may open the way to a sustainable use of the energy and to the reduction of the greenhouse gas emissions. The energy storage system is a fundamental block in many applications, from smart microgrids to electrified transportation systems, such as plug-in hybrid electric vehicles (PHEVs) and electric vehicles (EVs). The battery technologies used in these applications are growing fast to increase power and energy densities, battery lifetime and reduce costs, also extending the driving range of EVs [1]. The battery is usually equipped with a Battery Management System (BMS), that performs many functions: protection, monitoring, thermal and electric management, online fuel gauging and so forth.

At present, the superior performance of the lithium-ion (Li-ion) technology makes it the most attractive among the batteries, even if some safety issues, such as the fire susceptibility, and management issues are of concern. A battery with lithium iron phosphate $\mathrm{LiFePO}_{4}$ (briefly LFP) cathode [2] offers an excellent thermal stability, that means battery reliability and safety and a high number of cycles. It does not contains rare materials, with a positive impact on battery cost [3]. Although its power and energy density are not the best-achievable in the framework of Li-ion battery technology, its intrinsic safety, reliability and cost makes LFP technology one of the most promising solution for storage $[4,5]$.

LFP batteries are characterised by an almost constant Open-Circuit Voltage (OCV) when the stored charge is in the interval between $20 \%$ and $80 \%$ of its maximum value. In addition, the function mapping OCV as a function of the State-of-Charge $(\mathrm{SoC})$, i.e., the ratio between the charge stored in the battery and its maximum value, is not single-valued, but exhibits a pronounced hysteresis [6]. This phenomenon has a strong impact in BMS online fuel gauging. Indeed, the battery voltage, being hysteretic, cannot be used easily for Coulomb 
counting compensation, as often done for other Li-ion chemistries.

This phenomenon needs an accurate and computationally effective model, particularly suitable for online estimation in a BMS, where the computational resources are often limited by cost. To this end, this paper proposes the use of the classical Preisach model of hysteresis, often used for magnetic hysteresis, with a discretisation based on the so-called Everett function [7]. The paper achieves two important goals. First, the numerical implementation of the model proposed is computationally affordable for online SoC estimation in BMS. Additionally, the paper introduces an easily executable experimental battery characterisation procedure that allows the identification of the model. The model is validated with experiments representing various SoC "histories" of a $20 \mathrm{Ah}$ fresh LFP cell, tested with static and dynamic current profiles at a fixed temperature in one of our laboratories.

The paper is organised as follows. Section 2 introduces the nature of hysteresis in batteries and the related modelling attempts, motivating the particular Preisach approach proposed in this paper. Section 3 reports on experiments and test results, obtained in our laboratories, showing hysteresis in an LFP cell. Section 4 summarises the classical Preisach model, its application to batteries and its identification. Section 5 deals with the model validation, by comparing model simulations and experimental results. The limits of application of the model (ageing, temperature, current-rate and current dynamics) are discussed in Section 6 and, finally, some conclusions are drawn in Section 7.

\section{Battery hysteresis: state-of-the-art and modelling}

From a macroscopic point of view, OCV hysteresis in an LFP cell at given ageing state is characterised by the following properties. (i) It is a static phenomenon, as it remains after the battery current is switched off, even for a time exceeding the typical time constants of mass transport inside the electrodes. (ii) It is considered to be rate-independent, which means that it depends on the $\mathrm{SoC}$ history but not on the speed (battery current rate) with which SoC is changed. 
The rate-independence is matched with good approximation at least for low current rates, as shown in [8]. (iii) It exhibits sub-hysteresis loops included into a major one. Such properties match the ones exhibited by Nickel-Metal-Hydride (NiMH) batteries [9], deeply studied in the literature, where the open circuit potential of the nickel electrode shows a significant hysteresis [10-12].

An example of hysteretic behaviour experimentally measured on a $20 \mathrm{Ah}$ LFP cell at $298 \mathrm{~K}$ is given in Figure 1, where the full charge/discharge curves show a pronounced hysteresis (major loop).

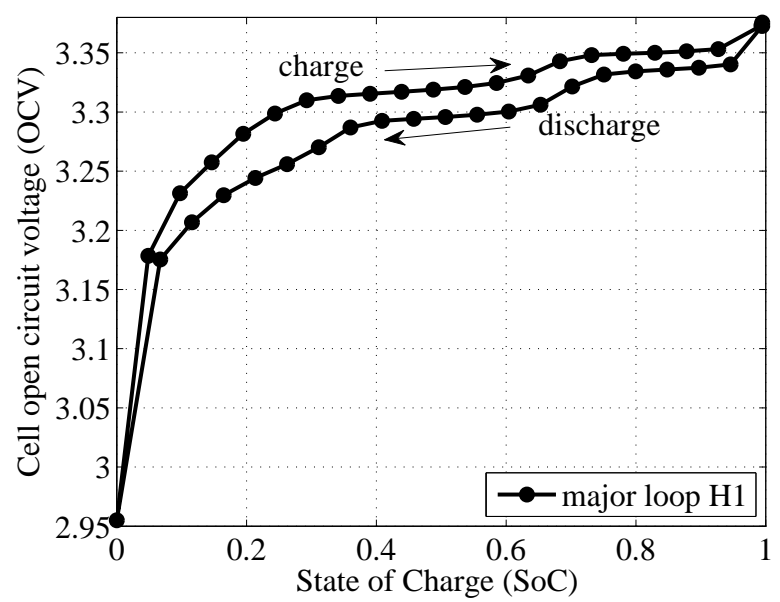

Figure 1: Open-Circuit Voltage vs State-of-Charge characteristic (hysteresis major loop) measured on a LFP $20 \mathrm{Ah}$ cell at $298 \mathrm{~K}$. The relaxation time for the OCV measurement is $1 \mathrm{~h}$.

Hysteresis in lithium-ion (Li-ion) batteries can be ascribed to thermodynamic entropic effects, mechanical stresses, and microscopic distortions within the active electrode materials during lithium insertion or extraction [13]. Evidence of hysteretic OCV behaviour has been reported for various anode and cathode materials. For instance, pronounced hysteresis effects have been observed in high temperature lithium insertion in hydrogen-containing carbons as a cathode material [14], as well as in innovative anode materials, such as NiO-graphene hybrid [15] and silicon oxycarbide (SiCO) [16]. The two-phase transition process leading to macroscopic OCV hysteresis, and the OCV recov- 
ery effects lasting for several minutes/hours after a current load is interrupted, are described for LFP batteries in [13], [17]. From a microscopic point of view, a thermodynamic consistent many-particle description of the electrode, based on the theory of many particle systems (ensembles of interconnected storage particles), is proposed in [18-20]. Such a model exhibits non-monotone behaviour leading to transitions between two coexisting phases and then to hysteresis.

Besides its physical origins, hysteresis is a phenomenon that must be considered in a large variety of battery applications, such as fast charging [21] and SoC estimation [22]. The availability of a reliable hysteresis model improves the accuracy of the algorithms for SoC estimation. The model should be simple for an easy implementation in embedded systems for advanced battery management. Therefore, large efforts have been directed to battery hysteresis modelling. As far as NiMH batteries are concerned, models first-principles equations [23], or the Nernst equation, including the entropy of reaction influence and an empirical expression to capture the salient features associated with voltage hysteresis $[10,24]$ are adopted. A multilayer model for nickel active materials with significant deviations from Nernst model is proposed in [25], a circuit approach with an RC "hysteretic" branch, based on an improved Takacs model [26], is used in $[27,28]$, where additional polynomial functions are employed to fit experimental data.

One of the models most used at the macroscopic/circuit level for Li-ion batteries is the One State Hysteresis (OSH) model [29], based on an approach adopted for magnetic materials [30]. The basic idea is that the major loop acts as a forcing term for a relaxation equation containing the signum operator. The model is simple and easy to apply. A circuit interpretation of this model is given in [31]. Similar signum-based models have been used in [32, 33] for online lead-acid state estimation. A simpler approach is adopted in [34], where the LFP battery hysteresis is modelled including two SoC-dependent OCV sources, one for the charging and one for discharging current, selected through two ideal diodes. Some other models have been proposed in the literature. The model in [35] is aimed at improving transient response with a hysteretic exponential 
term. Dynamic models to represent OCV as an output of a state-space model are proposed in $[36,37]$. Finally, the Jiles-Atherton model, identified by a neural network, describes the battery hysteretic characteristic in [38].

Hysteresis is a phenomenon deeply studied in magnetics and the paradigm of magnetic hysteresis modelling is the Preisach model. It was originally proposed by Preisach [39] in 1935 and later formalised in a general way [7, 40, 41] to take into account the similarity of hysteretic behaviours in different fields. Therefore, the application of the classical Preisach model to describe LFP battery hysteresis appears quite natural. An example of the use of the Preisach model to NiMH battery is reported in [42], where OCV is chosen as independent variable. This choice is not appropriate for LFP batteries, as they show a very flat SoC-OCV characteristic. The not natural discretisation of the Preisach operator that leads to a cumbersome identification based on a long training is another drawback of [42]. A very preliminary attempt to model lithium-ion batteries hysteresis with the Preisach approach is reported in [43], where the density function of the Preisach operator is assumed to be an a priori known function. However, this assumption is not general, very restrictive, and is combined with a non trivial neural network-based parameters identification procedure. Ref. [43] reports the application of the method only to the description of major loops and no validation is provided in the case of an arbitrary evolution of the battery SoC. Therefore, further work is necessary to obtain an effective and validated implementation of the Preisach model for hysteresis in LFP batteries. Our first attempt is described in [44], where a way to avoid any a priori choice of the Preisach density function was proposed.

In this paper, we apply the classical Preisach model for the static modelling of a fresh LFP cell SoC-OCV characteristic at fixed temperature, overcoming the intrinsic limitations and drawbacks highlighted in $[42,43]$ and extending the preliminary results obtained in [44]. The particular features of this approach are that SoC, rather than OCV, is assumed as independent variable of the model and that the Preisach operator is discretised by means of the so-called Everett function [7]. Two important goals are achieved: (i) a well-defined, easily exe- 
cutable and repeatable experimental procedure to identify the Everett function, and thus the Preisach operator, avoiding any heuristic approach to parameter/operator identification or any training is proposed; (ii) a computationally affordable model, suitable for online SoC estimation in BMS, where the available computational resources are limited by cost constraints is obtained. The model is validated with experiments representing various SoC "histories" of a 20 Ah fresh LFP cell, tested with static and dynamic current profiles at a fixed temperature in one of our laboratories.

\section{Experimental evidence of hysteresis on a LFP cell}

A brand-new $20 \mathrm{Ah}$ cell from GWL/Power is the LFP battery used for the experiments. Details of the cell are given in Table 1. The fresh cell was first conditioned at room temperature by ten full charge/discharge cycles after the delivery from the manufacturer. A Keithley SourceMeter Unit 2420 provides the battery supply/load, as well as accurate voltage and current measurements. All the tests are performed in a Binder MK35 thermal chamber that keeps the temperature at $298 \mathrm{~K}$. Therefore, the hysteresis analysis presented below is made without considering possible effects due to ageing and temperature variations.

\subsection{Pulsed current test}

We follow the procedure described in [31] to obtain the relationship between OCV and SoC. After the 10 cycle initialisation, a Pulsed Current Test (PCT) is performed. The battery is subjected to a sequence of constant-current (CC) pulse steps with duration $t_{\mathrm{on}}=30 \mathrm{~min}$, separated by zero-current rest steps $\left(t_{\text {rest }}=1 \mathrm{~h}\right)$. The usual relaxation of the terminal voltage is observed during the pauses. The CC pulse steps are either charge or discharge steps with a current value of $2 \mathrm{~A}$, i.e. one tenth of the battery rated capacity expressed in ampere-hours. In fact, each pulse determines a $5 \%$ variation of the SoC.

When the voltage reaches the upper/lower cutoff value, a constant-voltage (CV) phase begins to continue injecting/extracting energy until the current 
Table 1: Details of the cell used.

\begin{tabular}{c|c}
\hline \hline $\begin{array}{c}\text { manufacturer } \\
\text { cell name }\end{array}$ & $\begin{array}{c}\text { GWL/Power } \\
\text { LFP High Power Cell }\end{array}$ \\
\hline negative electrode \\
positive electrode \\
electrolyte & LiFeMnPO $_{4}$ \\
nominal capacity & $\mathrm{LiPF}_{6}$ \\
nominal voltage & $20 \mathrm{Ah}$ \\
maximum continuous discharge current & $3.2 \mathrm{~V}$ \\
cell operating temperature & $60 \mathrm{~A}$ \\
charge cutoff voltage & $253-323 \mathrm{~K}$ \\
discharge cutoff voltage & $3.65 \mathrm{~V}$ \\
\hline \hline
\end{tabular}

falls below a fixed threshold. Here, the threshold is $10 \%$ of the CC-mode current. These conditions, based on both voltage and current measurements, define the SoC boundary states: full-charge $(\mathrm{SoC}=100 \%)$ and full-discharge states ( $\mathrm{SoC}=0 \%$ ), as well as their corresponding OCV values, OCV (100\%) and $\operatorname{OCV}(0 \%)$. Since a few tens of millivolts spreading of these values is observed in different experiments, we define as OCV extrema their average values $\mathrm{OCV}_{0 \%}^{(\mathrm{av})}$ and $\mathrm{OCV}_{100 \%}^{(\mathrm{av})}$, respectively.

Given the SoC variation due to each CC pulse, the corresponding OCV value is defined as the cell terminal voltage reached at the end of each $t_{\text {rest }}$ [17]. An example of a current pulse in a PCT and the following extraction of the OCV value is shown in Figure 2. The SoC value is determined by integrating the current samples with a trapezoidal integration rule, and normalising the charge to a reference capacity. Reset points for integration are the full-charge and full-discharge states, so that the actual capacity measured after the complete charge-discharge loop is used as the reference capacity value. Figure 3 shows the flow chart of the procedure used to extract the SoC-OCV curve from the PCT data. 


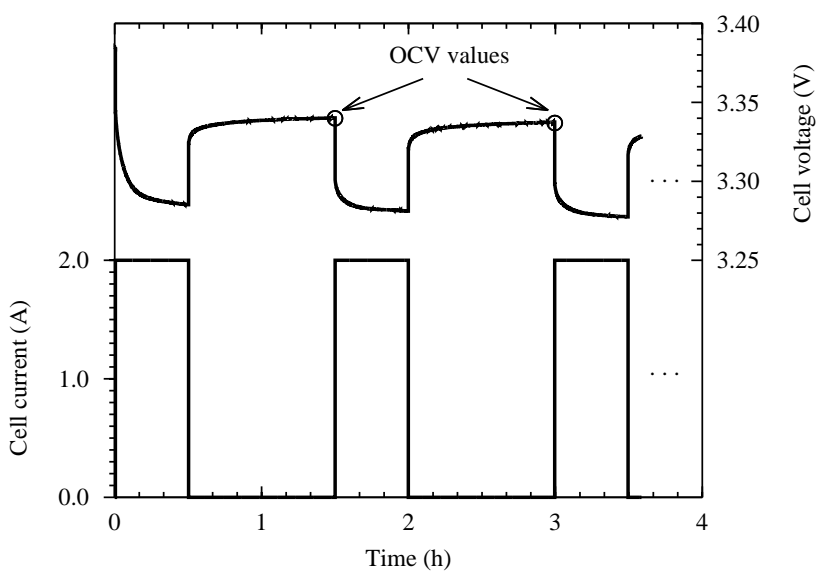

Figure 2: Example of a sequence of current pulses in a PCT and extraction of the OCV value at the end of the relaxation steps.

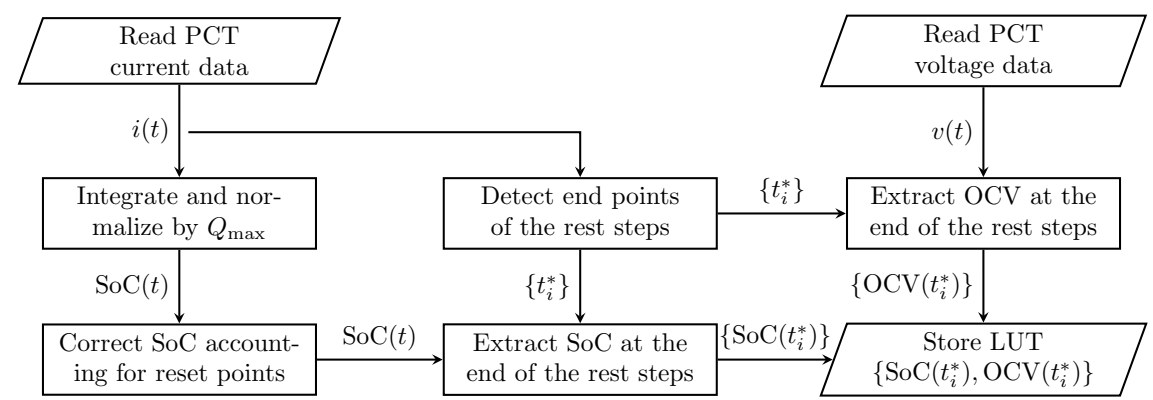

Figure 3: Flow chart describing the extraction procedure of the SoC-OCV characteristic starting from raw PCT data. Note that the set of values sampled at the end of the rest steps $t^{*}$ are represented in braces $\{\cdot\}$.

\subsection{Experimental results and evidence of hysteresis}

Five PCTs are carried out according to the aforementioned procedure. They are summarised in Table 2. The major hysteresis loop (H1) has already been shown in Figure 1. The charge and discharge curves are both rather flat, as expected, with two particularly flat zones around $\mathrm{SoC}=50 \%$ and $\mathrm{SoC}=75 \%$. The OCV varies less than $0.2 \mathrm{~V}$, a value as small as $6 \%$ of the $3.2 \mathrm{~V}$ nominal voltage, if we span the SoC from $10 \%$ to $90 \%$. 
Table 2: Description of the PCTs on a LFP 20 Ah cell. "Init." stands for "Initialisation phase".

\begin{tabular}{ccl}
\hline \hline label & description & SoC trajectory (\%) \\
\hline H1 & major loop & Init., 100-0-100 \\
H2 & history no. 2 & Init., 100-25-75-25-100 \\
H3 & history no. 3 & Init., 100-40-60-40-100 \\
H4 & history no. 4 & Init., 100-0-75-25-75-0-100 \\
H5 & FOR branches & Init., 100-0-90-0-...-10-0-100 \\
\hline \hline
\end{tabular}

Three SoC histories are run to investigate the battery behaviour inside the major loop, as described in Table 2 (H2-4). Figure 4 shows the related experimental results: the cell undergoes minor discharge/charge loops. SoC histories H2 and H3 do not reach the complete discharge, while $\mathrm{H} 4$ does it twice. The hysteresis is very similar to that found in magnetic materials, except for the shape and the orientation of the loops. The experiment H5 consists of loops of decreasing amplitude, all of which start from the full-discharge state. This experiment runs through the so-called First Order Reversal (FOR) branches. This experiment is the key for an easy and successful identification of the Preisach hysteresis model, as it will be shown in the following sections.

\section{Preisach modelling of hysteresis for an LFP cell}

\subsection{The Preisach model}

A brief summary of the Preisach model for hysteresis and its application to the LFP battery is given in this section. See [7] for a complete discussion on Preisach model implementation.

The Preisach hysteresis operator is defined as the superposition of ideal relay responses (depicted in Figure 5) by the following integral:

$$
y(t)=\int_{\alpha \geq \beta} \mu(\alpha, \beta) \hat{\gamma}_{\alpha \beta}\{x(t)\} d \alpha d \beta,
$$

where $x$ is the independent variable, $y$ is the dependent one, $\hat{\gamma}_{\alpha \beta}\{\cdot\}$ is the ideal relay operator shown in Figure 5 and $\mu$ is the density function of the relays 

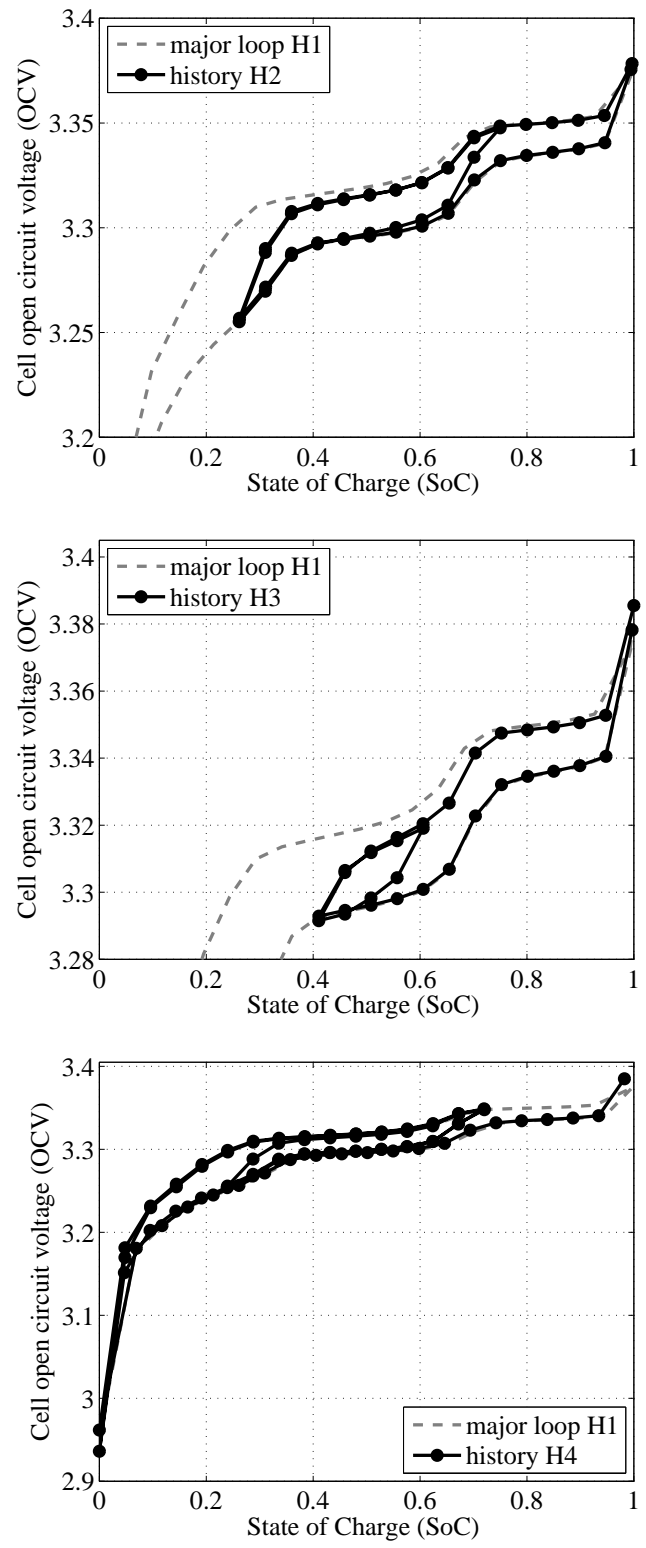

Figure 4: Experimental results related to the pulsed current tests H2-4, described in Table 2.

(also called Preisach function). The relay has two states: "up" $\left(\hat{\gamma}_{\alpha \beta}=+1\right)$ and "down" $\left(\hat{\gamma}_{\alpha \beta}=-1\right)$ and two switching thresholds: $\alpha$ is the switch-up threshold, and $\beta$ is the switch-down threshold. Each elemental relay is associated to the 
point $(\alpha, \beta)$. The integral (1) is calculated in the $\alpha-\beta$ plane for all the possible switching threshold couples $(\alpha, \beta)$, with $\alpha \geq \beta$. Being the thresholds bounded by the minimum and maximum values of $x(t)\left(x_{\min }\right.$ and $\left.x_{\max }\right)$, the integration domain is further restricted to the triangular domain $T$ called Preisach triangle (see Figure 6), yielding to:

$$
y(t)=\int_{T} \mu(\alpha, \beta) \hat{\gamma}_{\alpha \beta}\{x(t)\} d \alpha d \beta .
$$

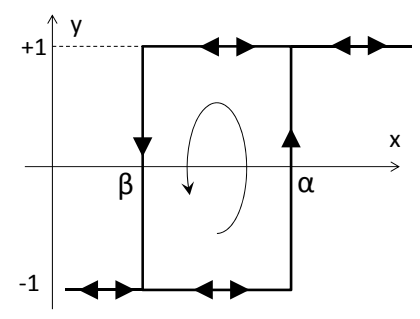

Figure 5: Ideal relay $\hat{\gamma}_{\alpha \beta}$.

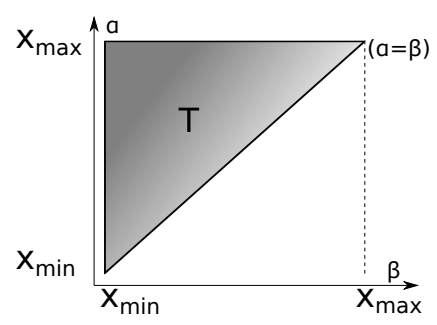

Figure 6: Preisach triangle.

The Preisach operator is characterised by two important properties, named wiping-out and congruency [7]. The wiping-out property characterises the memory of the physical system and states that the output of the Preisach operator is determined, at any time instant $t$, by a particular subset of input extrema occurred before the time $t$. The congruency states that all the minor loops of the $y$ variable corresponding to a back-and-forth variation of the input $x$ between the same extrema are congruent, i.e., exactly overlap if vertically shifted. It is often found that many physical systems showing hysteresis, including LFP batteries, fulfill the wiping-out property. Instead, the congruency property is only 
approximately satisfied, leading to possible mismatch between the responses of the model and the physical system.

Each time-history of the input-output variables can graphically be represented in the $\alpha-\beta$ plane by a line $L(t)$ composed of segments parallel to the $\alpha$ and $\beta$ axes [7], as shown in Figure 7. Each segment corresponds to a rising or falling part of the time history, between two extrema. $L(t)$ divides $T$ into two subdomains $S_{A}(t)$ and $S_{B}(t)$, where $A$ stands for "Above $L(t)$ " and $B$ for "Below $L(t)$ ". The relays are in the same state $\left(\gamma_{A}\right.$ or $\gamma_{B}$, respectively, with $\gamma_{B}=+1$ and $\left.\gamma_{A}=-1\right)$ in each subdomain. $L(t)$ evolves with time, but the last point is always located on the $\alpha=\beta$ edge of $T$. From a practical point of view, $L(t)$ can be coded as an array $\mathcal{L}$ storing the local input extrema that determine the vertices of $L(t)$.

Figure 7 shows with an example how $L(t)$ is constructed from the timedomain evolution of the input $x$ represented in Figure $7(\mathrm{a})$. The $x(t)$ extrema determine the vertices of $L(t)$, as highlighted by the horizontal dotted lines. Figure 8 shows that other input histories may lead to the same $L(t)$, as they are all characterised by the same extrema. Figure 8(c) shows a particular case where two local extrema (those not marked) are wiped-out by the successive ones. As a consequence, the previous behaviour of the function can fully be represented by the remaining extrema, that can thus be considered as the model memory state. The rules for the memory update based on the wiping-out property are extensively illustrated in [7].

The integral in (2) can be rewritten as

$$
y(t)=2 \int_{S_{B}(t)} \mu d \alpha d \beta-\int_{T} \mu d \alpha d \beta,
$$

in which its dependence on the memory state (the boundary of $S_{B}$ ) is made explicit. Equation (3) is the foundation for the practical calculation of the Preisach integral defined in (1). As the second integral in (3) is constant with time and is evaluated only once, the effective computation of the first integral in (3) leads to the computation of the output $y(t)$.

In summary, three steps characterises the model application: 


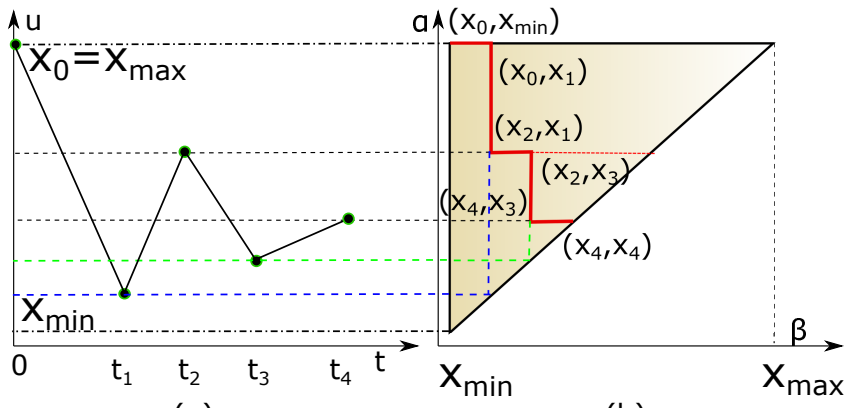

(a)

(b)

Figure 7: An example of the Preisach triangle $T$ partition into two subdomains (b) following to the input history plotted in (a). The partitioning interface is $L(t)$.

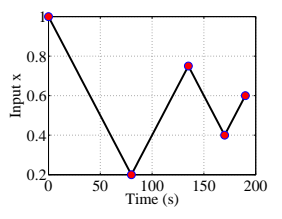

(a)

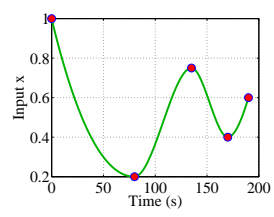

(b)

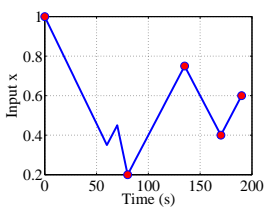

(c)

Figure 8: Three histories (a), (b) and (c) of the input $x(t)$ leading to the same hysteresis state and, thus, to the same interface $L(t)$. Red markers identify local extrema included into the memory state. (c) highlights that not all the local extrema are retained in the memory state, as they can be wiped-out by successive ones. 
i) the Preisach density $\mu$ (or a suitable auxiliary function) must be identified from experimental data.

ii) the boundary line $L(t)$ has to be found at each time step by recursively applying the wiping-out property, thus updating the memory state of the system.

iii) the output $y(t)$ is found by computing the integral of $\mu$ in the domain $S_{B}(t)$.

Keeping track of the input sequence allows the application of the wiping-out property by which only the significant extrema are stored in the system memory with low computational burden. Instead, the identification of the Preisach operator and the output calculation may be very tough issues to be solved. We use the so-called Everett function, identified with First-Order Reversal (FOR) branches data, to end up with a computationally efficient modeling procedure.

\subsection{First-order reversal branches and Everett function}

The procedure to extract the First-Order Reversal (FOR) branches is described below. A FOR branch $\mathcal{F}_{\alpha}$ is associated to the threshold $\alpha$. The input $x$ rises up to $\alpha$ from the "reset" state (every relay is in the "down" state, i.e., $S_{A}=T$ and $x=x_{\min }$ ). The output value in the $\alpha$ point is called $y_{\alpha}$ (inversion point). Then, $x$ is brought back to $x_{\min }$. The branch $\mathcal{F}_{\alpha}$ is drawn by taking the output value $y_{\alpha \beta}$ for any value $x=\beta$. The branch ends for $x=x_{\min }$, when $S_{A}=T$.

The Everett function is defined in [7] as:

$$
\mathcal{E}(\alpha, \beta)=\frac{y_{\alpha}-y_{\alpha \beta}}{2},
$$

which is half of the output variation along the FOR branch starting in $\alpha$. The Everett function is related to the Preisach function by the following integral:

$$
\mathcal{E}(\alpha, \beta)=\int_{T_{\alpha \beta}} \mu\left(\alpha^{\prime}, \beta^{\prime}\right) d \alpha^{\prime} d \beta^{\prime},
$$


where the integration domain $T_{\alpha \beta}$ is the triangle highlighted in Figure $9(\mathrm{a})$. We note that the integral on the triangle $T_{\alpha \beta}$ is a function of its upper-left corner and that $\mathcal{E}(\alpha, \beta)=0$ if $\alpha=\beta$ ( $T_{\alpha \beta}$ degenerates in a single point).

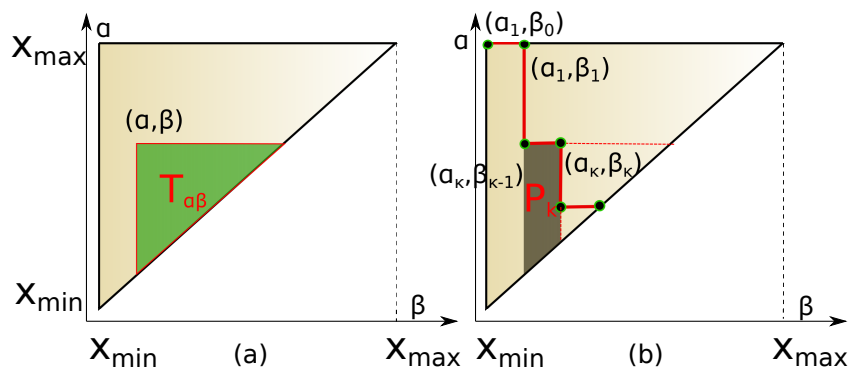

Figure 9: Geometrical interpretation of Preisach model. (a) Everett function $\mathcal{E}(\alpha, \beta)$ triangular integration domain. (b) Each $S_{B}$ is composed by trapezoidal strips $P_{k}$ of $T$.

The introduction of the Everett integral allows an easy computation of the first integral in (3). First of all, the integration domain $S_{B}$ is decomposed in $k$ trapezoidal vertical strips $P_{k}$, so that $S_{B}=\bigcup_{k} P_{k}$, as depicted in Figure 9(b). Then, each trapezius is expressed as combination of two triangles $P_{k}=\bigcup_{k}\left(T_{\alpha_{k} \beta_{k-1}} \backslash T_{\alpha_{k} \beta_{k}}\right)$. Remembering that the integral on a generic triangle $T_{\alpha \beta}$ is given by (5), the Preisach integral (3) becomes:

$$
y(t)=2 \sum_{k}\left[\mathcal{E}\left(\alpha_{k}, \beta_{k-1}\right)-\mathcal{E}\left(\alpha_{k}, \beta_{k}\right)\right]-\mathcal{E}_{0},
$$

where $\mathcal{E}_{0}=\mathcal{E}\left(x_{\max }, x_{\min }\right)=\int_{T} \mu d \alpha d \beta$. Two outstanding results comes from this procedure. First, the output is computed as a simple linear combination of the $\mathcal{E}$ values in the memory state points represented by the $L(t)$ vertices. Second, it is not necessary to identify the Preisach density $\mu$, as the identification of $\mathcal{E}$ in the domain $T$ is sufficient to apply the model. The identification of $\mathcal{E}$ is easy and is defined by a reliable and repeatable procedure based on the FOR branch experimental data.

\subsection{Application of the Preisach model to LFP batteries}

Our aim is now to apply the Preisach model to an LFP cell. We assume the $\mathrm{SoC}$ as the independent variable, so that $x=\mathrm{SoC}$ and $y=\mathrm{OCV}$ in the 
model, the opposite choice with respect to [42], as it seems to us more easy to control SoC rather than OCV. Thus, the Everett function has dimensions of volt. Choosing SoC as independent variable makes the hysteresis loop orientation in LFP batteries clockwise $(\mathrm{CW})$. Instead, the hysteresis loops modeled by the Preisach theory coming from magnetic materials are counter-clockwise (CCW). Although not intuitive, it is possible to keep the formalism introduced above, and thus to model also a CW hysteretic response, as the superposition of $\mathrm{CCW}$-oriented ideal relay contributions. The key point is to assume the density function $\mu$ appearing in the Preisach integral as the sum of a negative regular term (to account for the $\mathrm{CW}$ orientation of the loops), and an impulsive term located on the diagonal edge of $T$, which acts as a reversible term in the hysteretic output $[45,46]$. With this assumption, the theory applies to CW hysteresis also, and the results described by (6) is valid, i.e. the computation of the hysteretic variable is possible from the knowledge of the Everett function.

\subsection{Preisach model identification in LFP batteries}

As stated above, the application of the Preisach hysteresis model to LFP batteries is made easy by the identification of the Everett function (which completely specify the model) along some FOR branches. The experimental PCT cell characterisation H5 already described in Table 2 is carried out for ten FOR branches. The number of FOR branches explored comes from the trade-off between branch resolution and experiment duration. The experimental points are located in $T$ along ten horizontal lines, one per FOR branch (see Figure 10). The $i$-th line is located at $\alpha=\alpha_{i}$, where $\alpha_{i}$ is the SoC value of the starting point of the $i$-th FOR branch. The abscissa $\beta_{i j}$ of the $j$-th point of the $i$-th branch is the actual $\mathrm{SoC}$ value in that FOR branch point. The experimental data set is approximately equally spaced, both along $\beta$ ( $\Delta \beta=5 \%$ in SoC) and along $\alpha(\Delta \alpha=10 \%$ in SoC). The PCT results are shown in Figure 11. It is worth noting that the branches are very close to each other, but they do not overlap.

The experiment $\mathrm{H} 5$ allows the computation of the value $\mathcal{E}_{i j}$ of the Everett 


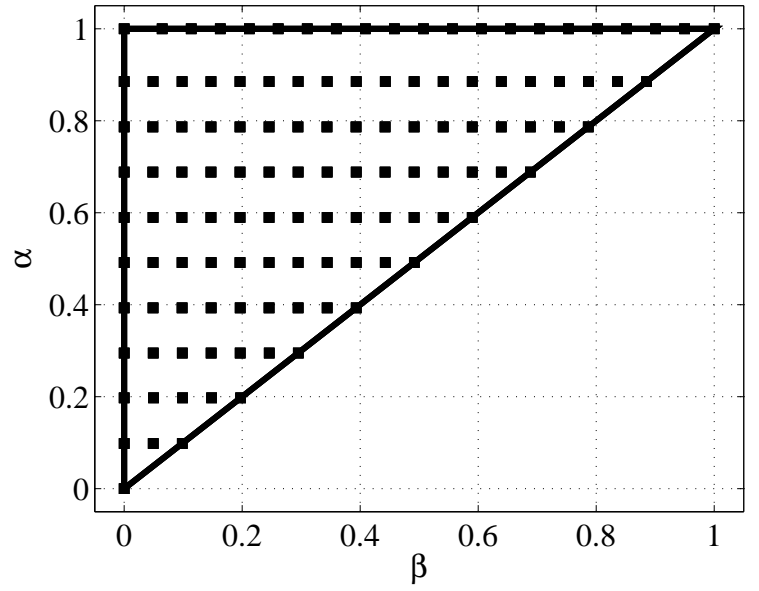

Figure 10: Evaluation points of the Everett function $\mathcal{E}$ as derived by the FOR branches experiment. Points are located along ten horizontal lines, corresponding to ten FOR branches.

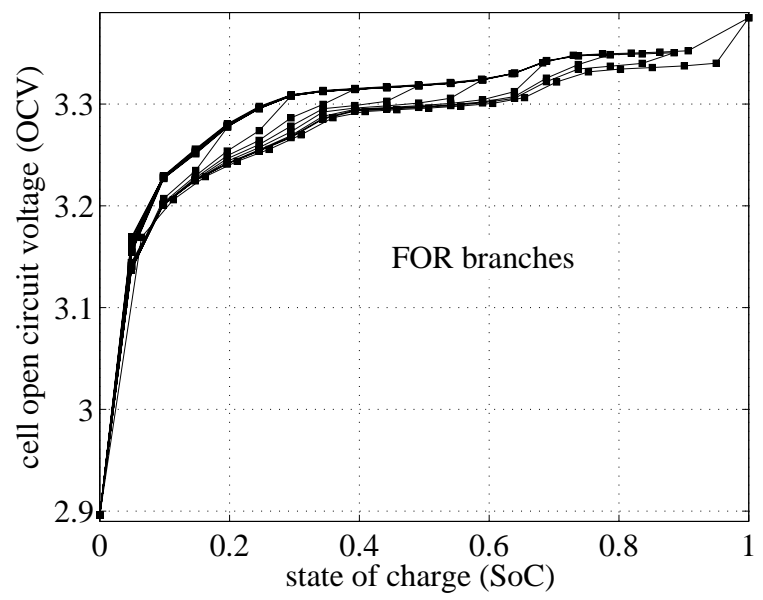

Figure 11: OCV-SoC characteristics along ten FOR branches of a 20 Ah LFP cell. 


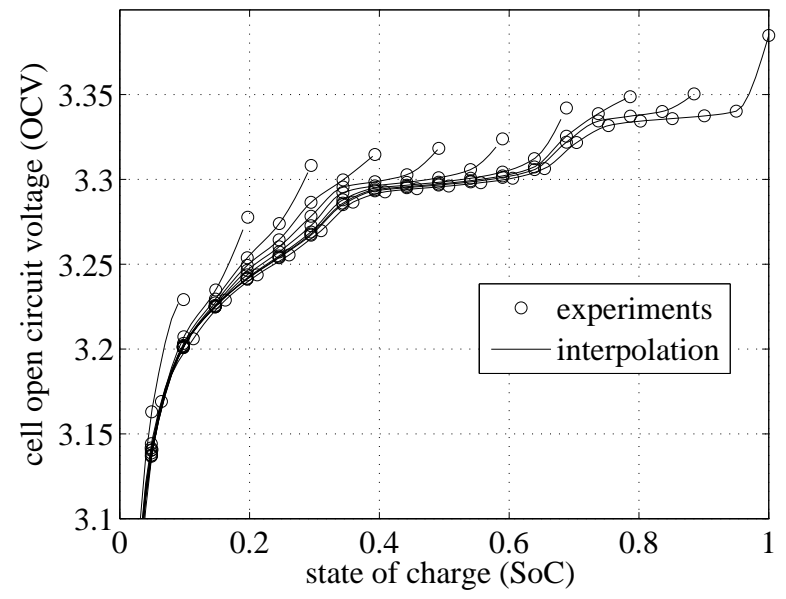

Figure 12: FOR branches for a 20 Ah LFP cell interploated on a $1 \%$ SoC grid from the experimental data.

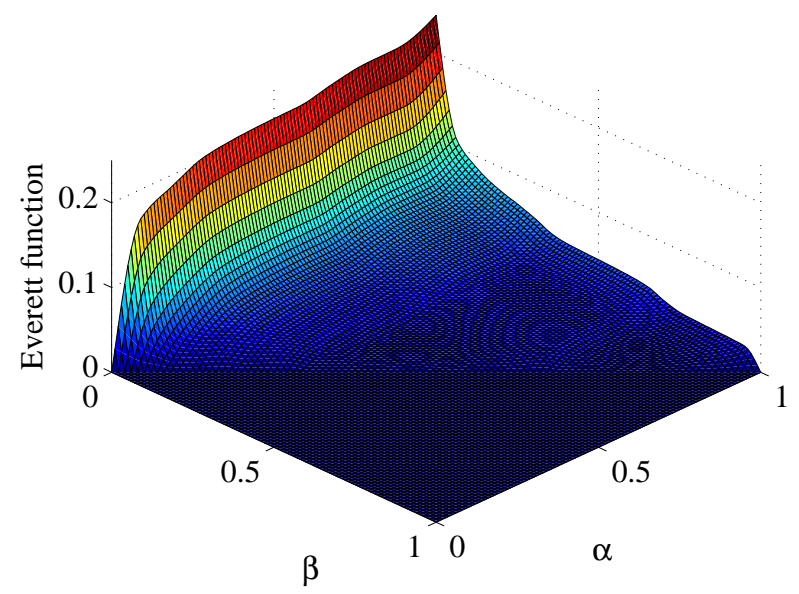

Figure 13: Everett function $\mathcal{E}$ in the whole Preisach triangle. 
function, according to (4), in all the experimental points $\left(\alpha_{i}, \beta_{i j}\right)$ in Figure 10. As the mesh of $T$ is rather coarse due to the limited number of measured branches, the Everett function has been reconstructed on a more fine and regular mesh according to the approach described in $[47,48]$. First, a one-dimensional (1D) interpolation of each FOR branch is performed, as shown in Figure 12, obtaining the solid lines from the experimental points. Then, another 1D interpolation in the orthogonal direction gives $\mathcal{E}$ along $\alpha$ for each fixed $\beta$.

Based on this finer two-dimensional discrete set, the Everett function values for any given point of $T$ can be calculated using a $2 \mathrm{D}$ interpolation, e.g. a piecewise linear function, or a piecewise constant function with less computational burden. A 2 D representation of the Everett function values $\mathcal{E}$ that identifies the Preisach model for our LFP battery is given in Figure 13.

\section{Results}

The model, identified by means of the experiment $\mathrm{H} 5$, is applied to the four SoC histories H1-4 in Table 2. The look-up table storing the Everett function values is based on a rectangular mesh $(\Delta \alpha=\Delta \beta=1 \%)$, and piecewise constant functions are used for the interpolation. The reconstruction algorithm is schematically reported in the flow diagram of Figure 14.

The results of the simulations will be compared to the experimental data and to another model found in the literature, the OSH model introduced in Section Section 5 and briefly recalled below.

\subsection{One-State Hysteresis model and identification}

In the OSH model, originally proposed by [29] and applied to LFP batteries in [31], the OCV is described by means of two auxiliary functions extracted by the hysteresis major loop. The first function $\mathrm{OCV}_{\mathrm{av}}(\mathrm{SoC})$ is the average of the charge and discharge OCV major loop branches. The second function $E(\mathrm{SoC})$ is half of the hysteresis interval in each SoC point, i.e. the value to be added/subtracted to the $\mathrm{OCV}_{\text {av }}$ to obtain the major loop charge/discharge 


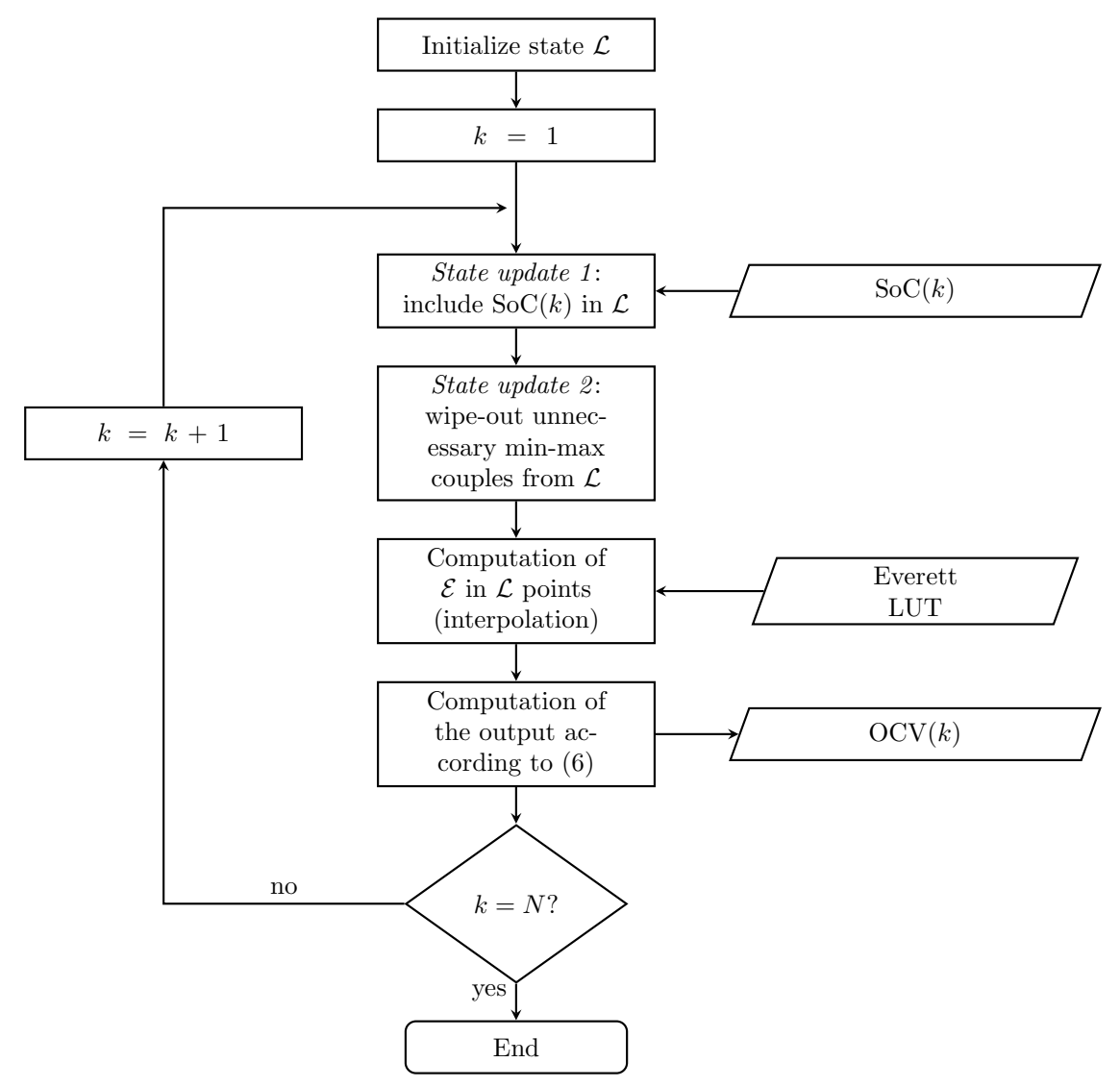

Figure 14: Procedure for the computation of the output according to Preisach model. Here, $\mathcal{L}$ is the hysteresis state, i.e. a vector that collects local maxima and minima of the input SoC that have not been wiped-out by upcoming extrema. $N$ is the number of SoC and OCV samples.

branches, respectively. These functions are plotted in Figure 15 for our LFP cell.

According to the OSH model, the overall OCV is expressed by $\mathrm{OCV}=\mathrm{OCV}_{\mathrm{av}}+$ $v_{\mathrm{H}}$, where $v_{\mathrm{H}}$ is the irreversible hysteretic term obtained by solving the following signum-based relaxation equation:

$$
\frac{d v_{\mathrm{H}}}{d \mathrm{SoC}}=-\operatorname{sign}(d \mathrm{SoC}) \gamma v_{\mathrm{H}}+\gamma E(\mathrm{SoC}) .
$$

The relaxation equation is forced by $E$, so that the major loop acts as an 

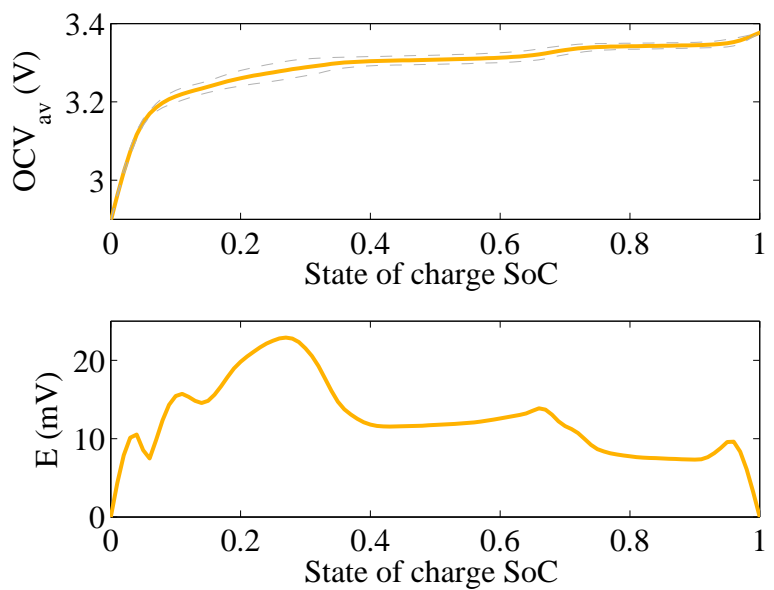

Figure 15: OSH model auxiliary functions. (a) Average OCV: $\mathrm{OCV}_{\mathrm{av}}(\mathrm{SoC})$ (solid line) between charge and discharge branches (dashed). (b) Maximum hysteresis $E$. These functions are extracted from the major loop obtained from the set of data H5.

attractor for the OCV. Here, $\gamma^{-1}$ is an adimensional constant (a "state-of-charge constant") working as the typical time constant of a time relaxation equation.

For a fair comparison, the OSH model is identified by the same set of experiments than the Preisach model, i.e. the H5 experimental data set. First of all the major loop branches are extracted and interpolated on a finer grid. Then, the functions $\mathrm{OCV}_{\mathrm{av}}(\mathrm{SoC})$ and $E(\mathrm{SoC})$ are derived. Finally, we find the optimal value of $\gamma$ that minimises the least-square error in the considered experiment H5. We obtain $\gamma_{\mathrm{opt}}=53.6$. It is worth noting that this value differs from the one reported in [31], showing a high sensitivity on the identification data set.

\subsection{Model validation and discussion}

Figures 16-19 collect the data coming from the simulations of both the models (Preisach, OSH) and the comparison with the experimental data. The relative error is computed with respect to a normalisation voltage $\triangle \mathrm{OCV}$ defined as the difference between the maximum and minimum OCV values, i.e., $\Delta \mathrm{OCV} \simeq 0.489 \mathrm{~V}$. The rms and peak errors are reported in a compact form in Table 3. 
Table 3: Relative errors expressed as percentage of $\triangle \mathrm{OCV}$.

\begin{tabular}{c|cc|cc|cc}
\hline & $\begin{array}{c}\text { Preisach } \\
\text { loop }\end{array}$ & $\begin{array}{c}\text { OSH } \\
(\mathrm{rms})\end{array}$ & $\begin{array}{c}\text { Preisach } \\
(\mathrm{rms})\end{array}$ & $\begin{array}{c}\text { OSH } \\
(\text { peak })\end{array}$ & $\begin{array}{c}\text { Preisach } \\
\left(\text { peak }^{*}\right)\end{array}$ & $\begin{array}{c}\text { OSH } \\
\left(\text { peak }^{*}\right)\end{array}$ \\
\hline \hline $\mathrm{H} 1$ & $2.52 \%$ & $2.59 \%$ & $12.1 \%$ & $13.99 \%$ & $1.75 \%$ & $1.72 \%$ \\
$\mathrm{H} 2$ & $1.13 \%$ & $1.23 \%$ & $2.79 \%$ & $1.70 \%$ & $2.79 \%$ & $1.70 \%$ \\
$\mathrm{H} 3$ & $0.57 \%$ & $0.95 \%$ & $1.18 \%$ & $1.03 \%$ & $1.18 \%$ & $1.03 \%$ \\
$\mathrm{H} 4$ & $2.39 \%$ & $2.66 \%$ & $13.44 \%$ & $15.44 \%$ & $2.33 \%$ & $3.16 \%$ \\
\hline \multicolumn{7}{c}{${ }^{*}$ computed in the interval $10 \% \leq \mathrm{SoC} \leq 90 \%}$.
\end{tabular}

The major loop H1 is characterised by an excellent agreement between simulated and experimental data except around the full-discharge state. The rms error is approximately equal to $2 \%$ and the peak error is around $12 \%$. The error values are similar for both the models. The rather large peak error is confined in a small region around the full-discharge point and is mainly due to the difference between the actual $\operatorname{OCV}(0 \%)$ in this experiment and the $\mathrm{OCV}_{0 \%}^{(\mathrm{av})}$ used in the identification procedure. Apart from this peak, the error is below $2 \%$ in the SoC range between $10 \%$ and $90 \%$ (see last columns of Table 3), showing a good modelling of the major loop.

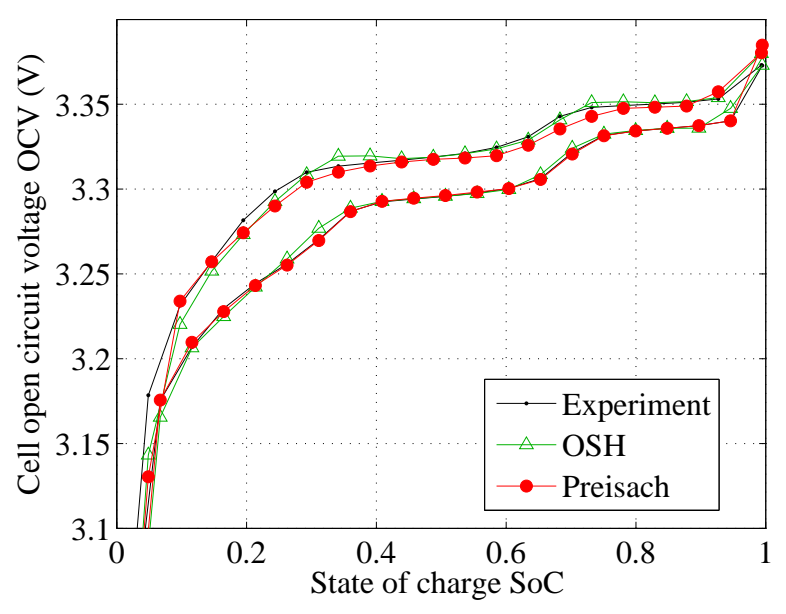

Figure 16: Model prediction compared with experimental results: loop H1. 


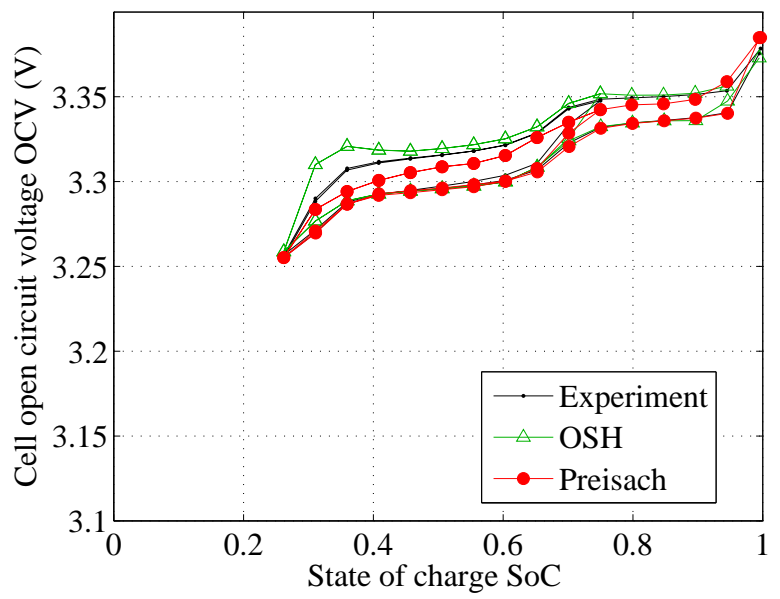

Figure 17: Model prediction compared with experimental results: loop H2.

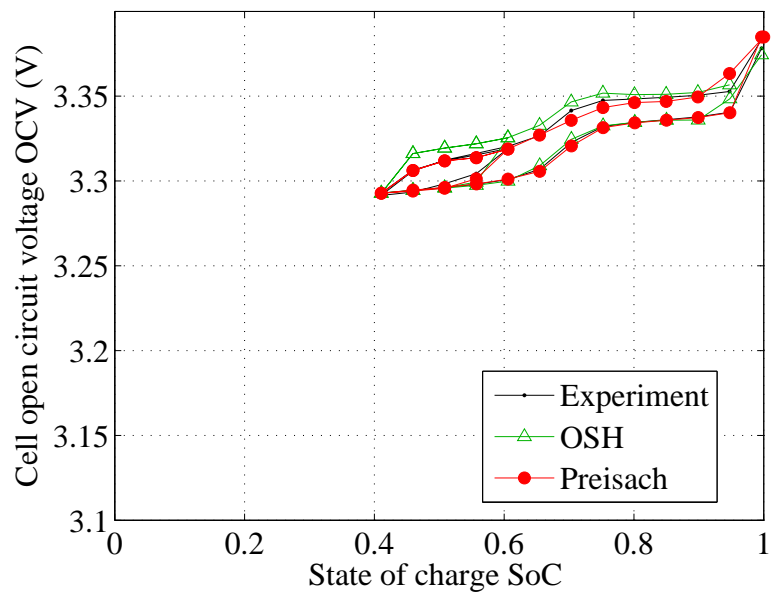

Figure 18: Model prediction compared with experimental results: loop H3. 


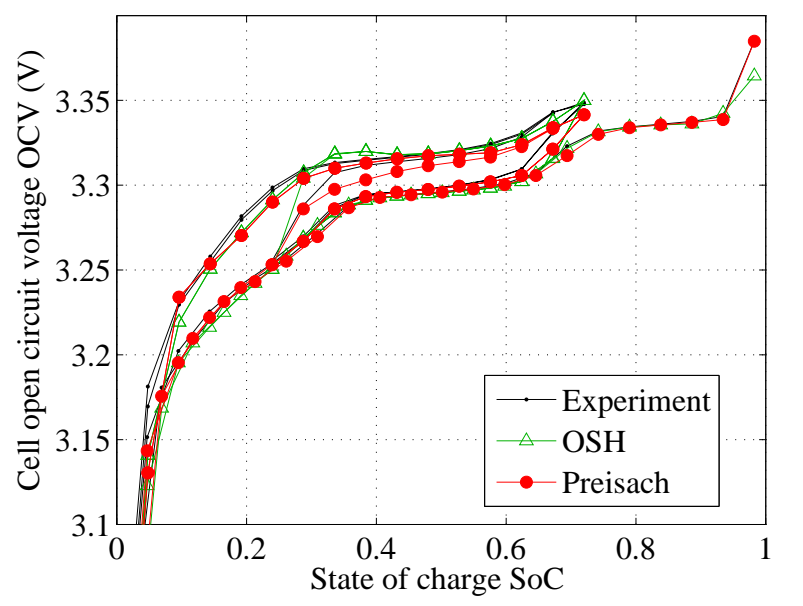

Figure 19: Model prediction compared with experimental results: loop H4.

Histories H2-H3, describing minor loops bounded in $25 \% \div 75 \%$ and $40 \% \div$ $60 \%$ SoC ranges, exhibit similar or even better performance. The rms error is around $1 \%$, while the peak error is below $3 \%$, as the full-discharge point is avoided. SoC history H4, in which the cell is completely discharged twice, is instead affected by a peak error comparable to that found in simulating H1. Finding a large peak error is reasonable, as the full-discharge region that is modelled with less accuracy is involved two times in this experiment. Similar numerical errors are achieved by OSH model in these three cases, even if a slight improvement of Preisach model with respect to OSH model is observed in terms of rms errors in all the cases analysed. In conclusion both the models reproduce the hysteretic cell behaviour rather accurately.

Besides the small improvements in the modeling accuracy, the Preisach approach stands for the valuable advantage of being based on a well-defined and repeatable cell characterisation procedure, that allows the identification of the hysteresis operator avoiding any heuristic approach, such as the one used in [31].

Both models have been identified here by the FOR branches data set that explores the entire hysteresis space in the OCV-SoC characteristic. On the one hand the OSH model appears to be very simple. On the other hand, the 
identification of the $\gamma$ parameter is highly sensitive to the used data set. This leads to a potential degradation of the OSH model capability of reproducing the hysteresis characteristics. Instead, Preisach model appears to be more robust from this point of view.

Referring again to the experimental results, it is worth noting that the errors are larger along the charge branches in all the cases considered, while discharge branches are modelled very accurately. We also notice that the model identification procedure was based on discharge-mode FOR branches (descending), so that the approximation is better along these branches rather than along the charge branches. The combined use of charge-mode (ascending) and dischargemode (descending) FOR branches during the operator identification could be a straightforward way to improve the model accuracy. This improvement is an additional advantage of the Preisach approach with respect to the OSH model.

To sum up, we can state that the hysteretic behaviour of the LFP cell is very well predicted by the Preisach model in all the experimental cases examined. Apart from the full-discharge region, the rms error values are very small, even lower than those found with the OSH model.

\section{Validity limit of the model and discussion}

As a matter of fact, the fidelity of the reconstruction of the Preisach model is proved for the case of a fresh cell working at fixed temperature. Moreover, the OCV is measured with PCTs with constant current values. Indeed, ageing, temperature and dynamic current profiles are not considered in this paper for the model validation. All these factors should be taken into account in order to use the proposed model in real cases, such as in an electric vehicle BMS for online SoC estimation. Let us discuss these points.

First of all, we would like to check whether the model can reproduce the OCV that follows the application of a dynamic current profile instead of a constant pulse. From a theoretical point of view, the model computes the OCV in response to a given $\mathrm{SoC}$ variations, no matter how that variation is obtained. 
So, the question is whether there is a dependence of the experimental hysteresis on the current profile. To investigate this, we stimulated the cell with dynamic current pulses, as shown in Figure 20. This pulse replaces the CC pulse used so far and it is equivalent to it, in the sense that the charge exchanged in each pulse is the same. The $t_{\text {rest }}$ between pulses is still kept at $1 \mathrm{~h}$. Figure 20 shows that, after an initial difference due to the last istantaneous current value, the relaxation voltages overlap and the measured OCV is the same. The responses to the same arbitrary SoC history realised with both the static and dynamic current profiles are reported in Figure 21. The results show that the static and dynamic responses overlap within $1 \%$ of $\triangle \mathrm{OCV}$, as it is shown in Figure 21. These values are comparable to the accuracy of the voltage measurement $(1.5 \mathrm{mV})$.

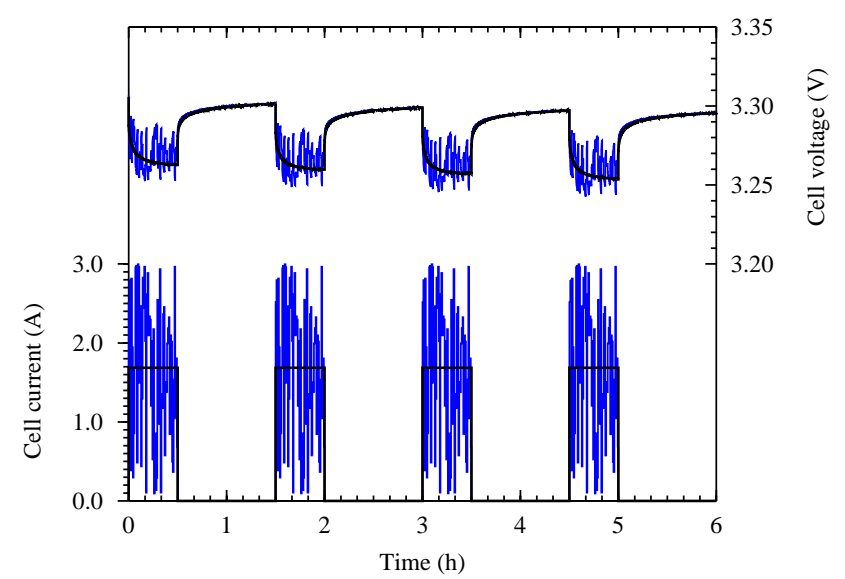

Figure 20: Example of PCTs with static (black lines) and dynamic (blue lines) excitation. The dc input current of the PCT is replaced with a dynamic profile. The voltage relaxations, apart from the very initial instants, tend to overlap.

The second issue to be discussed is the effect of the temperature $T_{b}$ that is not considered in this paper. Should the temperature have a significant effect on the hysteresis, the Preisach model implementation proposed here could address it by using a multidimensional LUT to reconstruct the OCV. In this case, the Everett table could be parametrised with respect to $T_{b}$, a solution often adopted in BMS for many other temperature-dependent battery parameters. A 

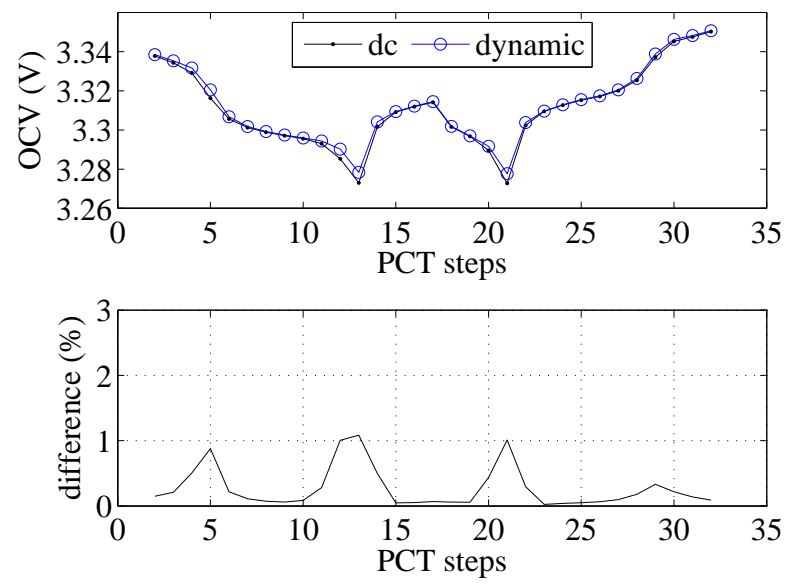

Figure 21: (a) OCV measured for the same SoC history with PCTs based on static (black) and dynamic (blue) input current excitations. The peak difference is below $1 \% \Delta \mathrm{OCV}$. (b) Relative difference between the static and the dynamic cases.

higher characterisation effort, i.e. the model identification with FOR branches at different temperatures, would solve the problem.

Finally, ageing might have significant impact on the size and shape of the hysteresis major and minor loops, even if the conclusive evidence of it is not reported in the literature yet. On the one hand, the idea of an Everett function LUT parameterised for ageing is appealing; on the other hand, ageing cannot be directly measured as it happens to temperature. In principle, if we take into account that the ageing dynamic is much longer than the typical mission time of a battery, a possible evolution of the model could be the periodical update of the Everett LUT based on the estimation of the current state-of-health of the battery. Further characterisation experiments on the hysteresis variation with ageing are however needed to address the issue.

\section{Conclusions}

The application of the Preisach model originally used in the magnetic material hysteresis characterisation to lithium-iron-phosphate (LFP) batteries, which 
show experimental evidence of a hysteretic behaviour between state-of-charge $(\mathrm{SoC})$ and open-circuit voltage $(\mathrm{OCV})$, is described in this paper. The main novelty of the paper is that the classical Preisach model for hysteresis applied to a LFP battery is identified by means of the Everett function, experimentally obtained from first-order reversal branch measurements. The hysteretic battery behaviours under various SoC histories are simulated by the identified Preisach model and compared to the experimental data obtained at constant temperature from a new LFP cell. It is found that the model accurately describes the measured hysteresis in the OCV of the LFP cell with rms errors of a few percent.

A comparison is carried out with another hysteresis modeling approach in LFP batteries, the one-state hysteresis model. Even if the reproduction errors are similar between the two approaches, the proposed technique exhibits two main advantages with respect to the other. First, the model is identified according to a well-defined experimental procedure to be performed on the battery, by which the Everett function values are extracted. This avoids any heuristic identification of the model parameters and reduces the model sensitivity to the identification procedure. Then, an easy and computationally affordable two-dimensional interpolation method for the Everett function, only sampled in a limited number of points, is applied. This means that the computation of the hysteresis voltage requires only linear combinations of the Everett function values calculated in particular inversion points of the battery SoC trajectory. This feature is very attractive for online applications where the battery SoC estimation is carried out in real time. A low model computational power is required in those cases, to make the estimation algorithm feasible and affordable on low-cost hardware platforms. Finally, the limits of application of the model are discussed, showing that the model correctly reproduces the hysteretic OCV even when the current pulses that excite the battery are not constant and are changed to a dynamic profile. The model is validated with fixed temperature experiments, but it is shown that temperature effects might be considered by adding one dimension to the LUT that stores the Everett function values, at the expense of a more extensive characterization procedure where the model 
identification is repeated at different temperatures.

\section{References}

[1] M. Armand, J.-M. Tarascon, Nature 451 (2008) 652-657.

[2] A. K. Padhi, K. S. Nanjundaswamy, J. B. Goodenough, Journal of the Electrochemical Society 144 (1997) 1188-1194.

[3] W. Zhang, Journal of Power Sources 196 (2011) 2962-2970.

[4] M. S. Whittingham, Proceedings of the IEEE 100 (2012) $1518-1534$.

[5] W. A. Paxton, Z. Zhong, T. Tsakalakos, Journal of Power Sources 275 (2015) 429-434.

[6] U. S. Kasavajjula, C. Wang, P. E. Arcea, Journal of the Electrochemical Society 155 (2008) A866-A874.

[7] I. D. Mayergoyz, Mathematical models of hysteresis and their applications, Academic Press, 2003.

[8] F. Baronti, W. Zamboni, R. Roncella, R. Saletti, G. Spagnuolo, in: Proceedings of the IEEE I2MTC 2015, Pisa, Italy, pp. 1-6.

[9] M. Thele, O. Bohlen, D. U. Sauer, E. Karden, Journal of Power Sources 175 (2008) 635-643.

[10] M. Verbrugge, E. Tate, Journal of Power Sources 126 (2004) 236-249.

[11] K. P. Ta, J. Newman, Journal of The Electrochemical Society 146 (1999) $2769-2779$.

[12] V. Srinivasan, J. W. Weidner, J. Newman, Journal of The Electrochemical Society 148 (2001) A969.

[13] M. A. Roscher, O. Bohlen, J. Vetter, International Journal of Electrochemistry 2011 (2011) $1-6$

[14] T. Zheng, J. R. Dahn, Journal of power sources 68 (1997) 201-203.

[15] Y. Mai, S. Shi, D. Zhang, Y. Lu, C. Gu, J. Tu, Journal of Power Sources 204 (2012) 155 $-161$

[16] D. Ahn, R. Raj, Journal of Power Sources 195 (2010) 3900-3906.

[17] M. A. Roscher, D. U. Sauer, Journal of Power Sources 196 (2011) 331-336. 
[18] W. Dreyer, C. Guhlke, R. Huth, Physica D: Nonlinear Phenomena 240 (2011) 1008-1019.

[19] W. Dreyer, C. Guhlke, M. Herrmann, Continuum Mechhanics and Thermodynamics 23 (2011) $211-231$.

[20] W. Dreyer, J. Jamnik, C. Guhlke, R. Huth, J. Moškon, M. Gaberšček, Nature materials 9 (2010) 448-453.

[21] S.-J. Huang, B.-G. Huang, F.-S. Pai, IEEE Transactions on Power Electronics 28 (2013) $1555-1562$.

[22] J. L. nad Joaquin Klee Barillas, C. Guenther, M. A. Danzer, Journal of Power Sources $230(2013) 244-250$.

[23] Y. Pan, V. Srinivasan, C. Wang, Journal of power sources 112 (2002) 298-306.

[24] M. W. Verbrugge, E. D. T. Jr, S. D. Sarbacker, B. J. Koch, Quasi-adaptive method for determining a battery's state of charge, 2002. US Patent 6,359,419.

[25] Y. Ota, Y. Hashimoto, Electrical Engineering in Japan 175 (2011) 1-7.

[26] J. Takacs, The International Journal for Computation and Mathematics in Electrical and Electronic Engineering (COMPEL) 20 (2001) 1002-1015.

[27] F. Xuyun, S. Zechang, in: Vehicle Power and Propulsion Conference, 2008. VPPC '08. IEEE, pp. 1-5.

[28] N. A. Windarko, J. Choi, in: Telecommunications Energy Conference, 2009. INTELEC 2009. 31st International, pp. 1-6.

[29] G. L. Plett, Journal of power sources 134 (2004) 262-276.

[30] D. Jiles, D. Atherton, Journal of magnetism and magnetic materials 61 (1986) 48-60.

[31] F. Baronti, W. Zamboni, N. Femia, R. Roncella, R. Saletti, in: IECON 2013 - 39th Annual Conference on IEEE Industrial Electronics Society, Vienna, pp. 6726-6731.

[32] S. Qiu, Z. Chen, M. Masrur, Y. Murphey, in: Industrial Electronics and Applications (ICIEA), 2011 6th IEEE Conference on, IEEE, pp. 184-189.

[33] A. Vasebi, M. Partovibakhsh, S. M. T. Bathaee, Journal of Power Sources 174 (2007) $30-40$.

[34] J. Kim, G.-S. Seo, C. Chun, B.-H. Cho, S. Lee, in: Electric Vehicle Conference (IEVC), 2012 IEEE International, pp. $1-5$. 
[35] A. Hussein, N. Kutkut, I. Batarseh, in: Applied Power Electronics Conference and Exposition (APEC), 2011 Twenty-Sixth Annual IEEE, IEEE, pp. 1790-1794.

[36] H. Zhang, M.-Y. Chow, in: IECON 2010-36th Annual Conference on IEEE Industrial Electronics Society, IEEE, pp. 1844-1849.

[37] H. R. Eichi, M.-Y. Chow, in: Energy Conversion Congress and Exposition (ECCE), 2012 IEEE, IEEE, pp. 4479-4486.

[38] M. Trapanese, V. Franzitta, A. Viola, in: Applied Power Electronics Conference and Exposition (APEC), 2013 Twenty-Eighth Annual IEEE, pp. 2773-2775.

[39] F. Preisach, Zeitschrift für physik 94 (1935) 277-302.

[40] M. Krasnoselskii, A. Pokrovskii, Systems with hysteresis, Springer Verlag, 1989.

[41] P. Krejci, Hysteresis, convexity and dissipation in hyperbolic equations, Gakkotosho, Tokyo, 1996.

[42] X. Tang, X. Zhang, B. Koch, D. Frisch, in: Prognostics and Health Management, 2008. PHM 2008. International Conference on, IEEE, pp. 1-12.

[43] V. Franzitta, A. Viola, M. Trapanese, Advanced Materials Research 622 (2013) 10991103.

[44] F. Baronti, N. Femia, R. Saletti, C. Visone, W. Zamboni, IEEE Transactions on Magnetics 50 (2014) 7300704

[45] I. D. Mayergoyz, P. Andrei, J Appl Phys 91 (2002) 7645-7647.

[46] C. Visone, W. Zamboni, IEEE Transaction on Magnetics 51 (2015) doi 10.1109/TMAG.2015.2434419.

[47] D. Davino, C. Natale, S. Pirozzi, C. Visone, Journal of Magnetism and Magnetic Materials 290-291 (2005) $1351-1354$.

[48] D. Davino, A. Giustiniani, C. Visone, IEEE Transactions on Magnetics 44 (2008) 862 865 . 